EPJ Web of Conferences 21, 07005 (2012)

DOI: $10.1051 /$ epjconf/20122107005

(C) Owned by the authors, published by EDP Sciences, 2012

\title{
On the unabridged 7D-folding structure of the optical model potential for nucleon-nucleus scattering
}

\author{
H. F. Arellano ${ }^{1, \mathrm{a}}$ and E. Bauge 2 \\ 1 Department of Physics - FCFM - University of Chile \\ Av. Blanco Encalada 2008, Santiago, Chile \\ 2 CEA/DAM/DIF, F-91297 Arpajon, France
}

\begin{abstract}
Microscopic optical model potentials for nucleon-nucleus collisions, based on density-dependent effective interactions, involve multi-dimensional integrals to account for the Fermi motion of the bound nucleons of the target. If a spherical matter distribution is assumed, each matrix element of the optical potential in momentum space requires the evaluation of seven-dimensional integrals. Here we describe the structure of these potentials and report results from their actual evaluation. Results for the scattering observables are compared with those based on alternative approximations, observing moderate sensitivity. These findings validate the use of simpler expressions to evaluate folding optical potentials, from which higher-order or unconventional effects in the effective interaction can safely be investigated.
\end{abstract}

\section{Introduction}

The optical model potential for nucleon-nucleus (NA) scattering has received considerable attention over the years. In addition to its conceptual interest from a fundamental point of view, the model has played an essential role as a tool for investigating and interpreting nuclear collisions and reactions. Its use not only provides a direct account for scattering observables in the collision process but also information about the distortion of the scattered waves, an important element in the study of inelastic processes. In the particular case of microscopic folding models for $N A$ scattering, the optical model potential becomes a one-body operator which relies on a basic link between the interaction of two nucleons in free space and an effective interaction which embodies, in principle, all correlations implicit in the NA many-body system. Only with the introduction of simplifying assumptions this link becomes feasible. Some of these simplifications come after truncations in the perturbative expansions for the effective interaction. Others emerge from treatment of its coordinate- and/or momentum-space structure of the effective interaction, simplifying its general structure and reducing the number degrees of freedom to deal with. Here we focus on the latter aspect, providing a complete account of the Fermi motion of the struck nucleon while retaining the exact momentum structure of the genuine effective interaction, as obtained from the Brueckner-Bethe-Goldstone (BBG) for infinite nuclear matter. This feature goes beyond all previous density-dependent approaches where the effective interaction is either localized [1-4] or the full momentum dependence implied by the basic collisions is simplified [5,6].

Microscopic optical model potentials for NA scattering have been widely applied over the years, covering diverse targets and over a wide energy range, featuring in many cases striking descriptions of the data. However, there are energies and targets where clear disagreement between the theory and the data is observed, particularly at beam energies below $70 \mathrm{MeV}$. In some cases a slight adjustment in the strength of the real and/or imaginary part of the calculated potential yield substantial improvements in the description of the data. From a theoretical point of view, however, these shortcoming have

\footnotetext{
a e-mail: arellano@dfi.uchile.cl
}

This is an Open Access article distributed under the terms of the Creative Commons Attribution-Noncommercial License 3.0, which permits unrestricted use, distribution, and reproduction in any noncommercial medium, provided the original work is properly cited. 


\section{EPJ Web of Conferences}

the potential to identify conventional or unconventional mechanisms which may be relevant in the collision process. Thus, in the context of folding models, it is of interest to investigate its unabridged realization, where a detailed treatment of the genuine effective interaction is accounted for. Once this limit is reached, the role of unconventional effects may be assessed more clearly. To illustrate with one example, it is well known that three-body forces do play an important role in the saturation properties of nuclear matter [7]. Actually with the appropriate calibration of these forces it is possible to account for the experimental saturation point. Hence, it becomes natural to ask whether such three-body forces become also relevant in scattering processes, and if an eventual improvement in the account for the data can be attributed to such forces.

The calculation of folding optical model potentials reported here, and more extensively in Ref. [8], is based on a general property that establishes that the intrinsic nuclear medium effects, namely those arising from the density dependence of the $g$ matrix, are dominantly confined in the nuclear surface due to its steepest gradient [9]. This property is an analytic consequence of the momentum- and coordinatespace structure of a two-body effective interaction spherically symmetric in the mean coordinate of interacting pair. As demonstrated in Ref. [9], any two-body operator can be expressed as a non-trivial sum of a translationally invariant term and another which is functionally-and exclusively-proportional to the gradient of a reduced in-medium operator. As a result, the optical potential in momentum space becomes the sum of medium-free (free $t$ matrix) and medium-dependent ( $g$ matrix) contributions, the latter depending exclusively on the variations of the effective interaction with respect to the mean coordinate.

\section{Framework}

In quite general terms, diverse formal expressions of the optical model potential for NA elastic scattering can be found in the literature [1]. Although all these many-body models may differ in the way the contact is made with the bare nucleon-nucleon $(N N)$ interaction, they all have the structure of a ground-state expectation value of a generalized two-body interaction. Thus, a general structure of the optical model potential for collisions of nucleons with kinetic energy $E$ off a composite $A$-nucleon target is given by the folding integral

$$
U\left(\boldsymbol{k}^{\prime}, \boldsymbol{k} ; E\right)=\int d \boldsymbol{p}^{\prime} d \boldsymbol{p}\left\langle\boldsymbol{k}^{\prime} \boldsymbol{p}^{\prime}|\hat{T}(E)| \boldsymbol{k} \boldsymbol{p}\right\rangle_{\mathcal{A}} \hat{\rho}\left(\boldsymbol{p}^{\prime}, \boldsymbol{p}\right),
$$

where the subscript $\mathcal{A}$ stands for antisymmetrization. Spin and isospin labels are omitted for simplicity. Here $\hat{\rho}\left(\boldsymbol{p}^{\prime}, \boldsymbol{p}\right)$ represents the target mixed density in momentum representation.

A simple but important consideration is that the two-body operator $\hat{T}$ can be equivalently represented in momentum or coordinate space. Its momentum representation is given by the matrix elements $\left\langle\boldsymbol{k}^{\prime} \boldsymbol{p}^{\prime}|\hat{T}(E)| \boldsymbol{k} \boldsymbol{p}\right\rangle$, whereas its coordinate-space counterpart is represented by $\left\langle\boldsymbol{r}^{\prime} \boldsymbol{s}^{\prime}|\hat{T}(E)| \boldsymbol{r} s\right\rangle$. These two elements relate to each other by means of simple Fourier transforms. Note that $r(s)$ represent the prior coordinates of the projectile (target nucleon), while the primed quantities refer to the post coordinates. These four vectors define the mean coordinate $z$ given by

$$
z=\frac{1}{4}\left(\boldsymbol{r}^{\prime}+\boldsymbol{s}^{\prime}+\boldsymbol{r}+\boldsymbol{s}\right) .
$$

As we shall see, this coordinate plays an important role in the way site-dependent properties are tracked in the system.

Following Ref. [9], the momentum-space representation of any two-body operator $\hat{T}$ can be cast in terms of a reduced interaction, $g_{z}$, in the form

$$
\left\langle\boldsymbol{k}^{\prime} \boldsymbol{p}^{\prime}|\hat{T}| \boldsymbol{k} \boldsymbol{p}\right\rangle=\int \frac{d \boldsymbol{z}}{(2 \pi)^{3}} e^{i \boldsymbol{z} \cdot(\boldsymbol{Q}-\boldsymbol{q})} g_{\boldsymbol{z}}\left(\boldsymbol{K}_{\|} ; \boldsymbol{b}^{\prime}, \boldsymbol{b}\right) .
$$

Here we denote $\boldsymbol{q}=\boldsymbol{k}-\boldsymbol{k}^{\prime}$, and $\boldsymbol{Q}=\boldsymbol{p}^{\prime}-\boldsymbol{p}$, accounting for the momentum transfer of the projectile and recoil of the target nucleon, respectively. Additionally, $\boldsymbol{K}=\left(\boldsymbol{k}+\boldsymbol{k}^{\prime}\right) / 2$, and $\boldsymbol{P}=\left(\boldsymbol{p}^{\prime}+\boldsymbol{p}\right) / 2$, representing the mean momentum of the projectile and struck nucleon, respectively. 


\section{$\mathrm{CNR} * 11$}

In terms of these momenta, the post and prior relative momenta become expressed as

$$
\begin{gathered}
\boldsymbol{b}^{\prime}=\frac{1}{2}\left(\boldsymbol{k}^{\prime}-\boldsymbol{p}^{\prime}\right)=\frac{1}{2}\left[\boldsymbol{K}-\boldsymbol{P}-\frac{1}{2}(\boldsymbol{q}+\boldsymbol{Q})\right], \\
\boldsymbol{b}=\frac{1}{2}(\boldsymbol{k}-\boldsymbol{p})=\frac{1}{2}\left[\boldsymbol{K}-\boldsymbol{P}+\frac{1}{2}(\boldsymbol{q}+\boldsymbol{Q})\right],
\end{gathered}
$$

respectively. In Eq. (2) $\boldsymbol{K}_{\|}=\boldsymbol{K}+\boldsymbol{P}$, interpreted as the current of the interacting nucleons, consisting on the sum of the prior and post mean momenta of the colliding pair. With these definitions the integrals on $\left(\boldsymbol{p}, \boldsymbol{p}^{\prime}\right)$ in Eq. (1), which sums over for the Fermi motion of the target nucleons, are accounted for by $(\boldsymbol{P}, \boldsymbol{Q})$, with $d \boldsymbol{p}^{\prime} d \boldsymbol{p}=d \boldsymbol{Q} d \boldsymbol{P}$.

Before using Eq. (2) for $\hat{T}$ in the general expression for the folding integral, it is interesting to note that if $g_{z}$ is invariant under translations (i.e. $g_{z} \equiv g_{0}$, independ of the mean coordinate $z$ ), then

$$
\left\langle\boldsymbol{k}^{\prime} \boldsymbol{p}^{\prime}|\hat{T}| \boldsymbol{k} \boldsymbol{p}\right\rangle=\delta(\boldsymbol{Q}-\boldsymbol{q}) g_{0}\left(\boldsymbol{K}_{\|} ; \boldsymbol{b}^{\prime}, \boldsymbol{b}\right) .
$$

Therefore, $\boldsymbol{Q}=\boldsymbol{q}$, implying momentum conservation as expected.

The reduced interaction, $g_{z}$, is modeled here as the off-shell $g$ matrix for infinite symmetric nuclear matter in the BBG theory. At each site $z$ in the nucleus one identifies the isoscalar density $\rho(z)$ at which the $g$ matrix is evaluated. Hence, the realization of the unabridged optical potential requires off-shell $g$ matrices over a wide range of densities. In the case of spherically symmetric matter distributions $[\rho(z) \rightarrow \rho(z)]$, the $z$ integral in Eq. (2) can be split in such a way that its asymptotic (medium-free) structure becomes isolated from the $z$-dependent term. Accordingly [9]

$$
\left\langle\boldsymbol{k}^{\prime} \boldsymbol{p}^{\prime}|\hat{T}| \boldsymbol{k} \boldsymbol{p}\right\rangle=\delta(\boldsymbol{Q}-\boldsymbol{q}) t\left(\boldsymbol{K}_{\|} ; \boldsymbol{b}^{\prime}, \boldsymbol{b}\right)-\frac{1}{6 \pi^{2}} \int_{0}^{\infty} z^{3} d z S(z|\boldsymbol{Q}-\boldsymbol{q}|) \frac{\partial g_{z}}{\partial z},
$$

where the momentum dependence of $\left(\partial g_{z} / \partial z\right)$ on $\boldsymbol{K}_{\|}, \boldsymbol{b}^{\prime}$ and $\boldsymbol{b}$ is implicit. Here $S(x)=3 j_{1}(x) / x$. A direct consequence of the result above is that the optical potential becomes the sum of two terms, $U=U_{0}+U_{1}$, with

$$
\begin{aligned}
& U_{0}\left(\boldsymbol{k}^{\prime}, \boldsymbol{k} ; E\right)=\int d \boldsymbol{P} \hat{\rho}(\boldsymbol{q} ; \boldsymbol{P}) t\left(\boldsymbol{K}_{\|} ; \boldsymbol{b}^{\prime}, \boldsymbol{b}\right) ; \\
& U_{1}\left(\boldsymbol{k}^{\prime}, \boldsymbol{k} ; E\right)=\frac{-1}{6 \pi^{2}} \int_{0}^{\infty} z^{3} d z \int d \boldsymbol{Q} d \boldsymbol{P} \hat{\rho}(\boldsymbol{Q} ; \boldsymbol{P}) \mathcal{S}(z|\boldsymbol{Q}-\boldsymbol{q}|) \frac{\partial g_{z}}{\partial z} .
\end{aligned}
$$

Here we denote

$$
\hat{\rho}(\boldsymbol{Q} ; \boldsymbol{P}) \equiv \hat{\rho}\left(\boldsymbol{P}+\frac{1}{2} \boldsymbol{Q}, \boldsymbol{P}-\frac{1}{2} \boldsymbol{Q}\right) .
$$

The term $U_{0}$ depends exclusively on the reduced matrix in free space. This contribution is referred as full-folding optical potential, with its first realizations in momentum space reported in the early nineties [1]. For this particular contribution the effective interaction becomes the off-shell scattering matrix in the framework of the Lippmann-Schwinger equation.

The second term, $U_{1}$, depends on the gradient with respect to the mean coordinate of the $g$ matrix. It involves a seven-fold integral, one of them along the radial coordinate $z$ and the other $\operatorname{six}\left(d^{3} P d^{3} Q\right)$ to account for the Fermi motion of the struck nucleon. Since $g=g[\rho(z)]$, then $\partial g / \partial z=(\delta g / \delta \rho)(\partial \rho / \partial z)$. This structure confines the radial integration to regions where the density varies most, i.e. the nuclear surface. The resulting optical potentials become non local, and their treatment to obtain the scattering observables can be seen in Ref. [10].

We have been able to evaluate optical model potentials in its unabridged form for proton elastic scattering at energies above $30 \mathrm{MeV}$. Its results for the scattering observables are compared with the following two approximations:

a) The $\delta g$-folding.- introduced in Ref. [6], where the dependence of the $g$ matrix on $Q$, the recoil of the target nucleon, is neglected. This is implemented by setting $\boldsymbol{Q}=\boldsymbol{q}$, the momentum transfer of the projectile. Considering that $Q=p^{\prime}-\boldsymbol{p}$, and $\boldsymbol{q}=\boldsymbol{k}-\boldsymbol{k}^{\prime}$, then this approximation is equivalent to set

$$
\boldsymbol{k}^{\prime}+\boldsymbol{p}^{\prime}=\boldsymbol{k}+\boldsymbol{p},
$$

i.e. momentum conservation. Therefore, the $\delta g$-folding evaluates the $g$ matrix as if the momentum was conserved in the two-body interaction, in contrast with the unabridged folding. 


\section{EPJ Web of Conferences}

b) The ABL-folding.- Here the optical potential takes the form introduced by Arellano, Brieva and Love (ABL) [5]. To obtain this form one simply makes use of the Slater approximation for the mixed density, starting from the expression for the optical potential within the $\delta g$-folding. Hence,

$$
U_{A B L}\left(\boldsymbol{k}^{\prime}, \boldsymbol{k} ; E\right)=\int d \boldsymbol{z} e^{i\left(\boldsymbol{k}^{\prime}-\boldsymbol{k}\right) \cdot \boldsymbol{z}} \rho(\boldsymbol{z}) \bar{g}_{z}\left(\boldsymbol{k}^{\prime}, \boldsymbol{k}\right),
$$

where $\rho(z)$ is the point density at the mean coordinate $z$ and $\bar{g}$ represents an off-shell Fermiaveraged amplitude at the local target density $\rho(\boldsymbol{Z})$. More explicitly,

$$
\bar{g}_{z}\left(\boldsymbol{k}^{\prime}, \boldsymbol{k}\right)=\frac{3}{4 \pi \hat{k}_{F}^{3}} \int \Theta(\hat{k}-|\boldsymbol{P}|) g\left(\boldsymbol{b}^{\prime}, \boldsymbol{b} ; E\right) d \boldsymbol{P} .
$$

Here at each site $z$ one evaluates $\bar{g}$ based on BBG $g$ matrices at the local Fermi momentum $k_{F}$, where

$$
\rho(z)=\frac{2}{3 \pi^{2}} k_{F}^{3} .
$$

Among the advantages of this approximation is the fact that one needs only the radial density of the nucleus, together with the fact that it is rather brief to evaluate.

\section{Applications}

We have performed calculations of the 7D-folding optical potentials and the two approximations described above for proton scattering from ${ }^{16} \mathrm{O}$ and ${ }^{90} \mathrm{Zr}$, at beam energies above $30 \mathrm{MeV}$. These calculations are based on $g$ matrices using the Paris $N N$ potential [13]. The $g$ matrices are obtained solving the BBG integral equation, once achieved selfconsistency in the auxiliary fields within the continuous choice. The calculated (off-shell) $g$ matrices are tabulated at 15 different densities. For the mixed density we use Hartree-Fock solutions which give adequate account of the charge r.m.s radius. The folding integrals are performed by means of Gaussian quadratures. It is worth noting that the computing time needed to evaluate these potentials go as 500:20:1, with the unabridged taking longer time and the ABL the fastest. The calculated optical potentials, in momentum space, are then passed to coordinate space in order to solve the Lippmann-Schwinger equation for the wavefunction [10].

In Figs. 1 and 2 we present results for the scattering observables for proton scattering from ${ }^{16} \mathrm{O}$ and ${ }^{90} \mathrm{Zr}$, respectively, based on the three approaches described above. In the upper frame we show the differential cross section $d \sigma / d \Omega$, whereas in the lower frames we show the analyzing power $A_{y}$. Results for the 7D-folding potential are denoted with solid curves; for the $\delta g$-folding we use dashed curves and the ABL results are shown with dotted curves. All of them are based on the Paris potential. We note that the differences among them are quite moderate, particularly in the cross section. Such is not the case for $A_{y}$, where differences of the order of 0.3 appear at back angles. Still, we conclude that all three approaches yield comparable results.

In order to gauge these differences relative to other effects, we have calculated optical potentials within the ABL approach using the Argonne $v_{18}[14] N N$ potential. The corresponding results are also shown in Figs. 1 and 2, but using solid red curves. We note that the use of this $N N$ potential yield scattering observables distinguishable from the ones based on the Paris potential. We have to keep in mind that these two $N N$ models are not on-shell equivalent. Actually, they lead to different phase shifts in some channels. Nevertheless, both $N N$ models are among the most complete current non-relativistic descriptions of the $N N$ interactions, setting the limits of our current understanding of the force between two nucleons in free space. These limitations imply uncertainties in the calculated cross sections which lay between the red solid curves and the dotted ones, both within the ABL approach.

\section{Summary and conclusions}

We have been able to evaluate for the first time the seven-dimensional folding integral for the optical model potential, retaining the genuine structure of the $N N$ effective interaction. The reduced interaction 


\section{$\mathrm{CNR} * 11$}

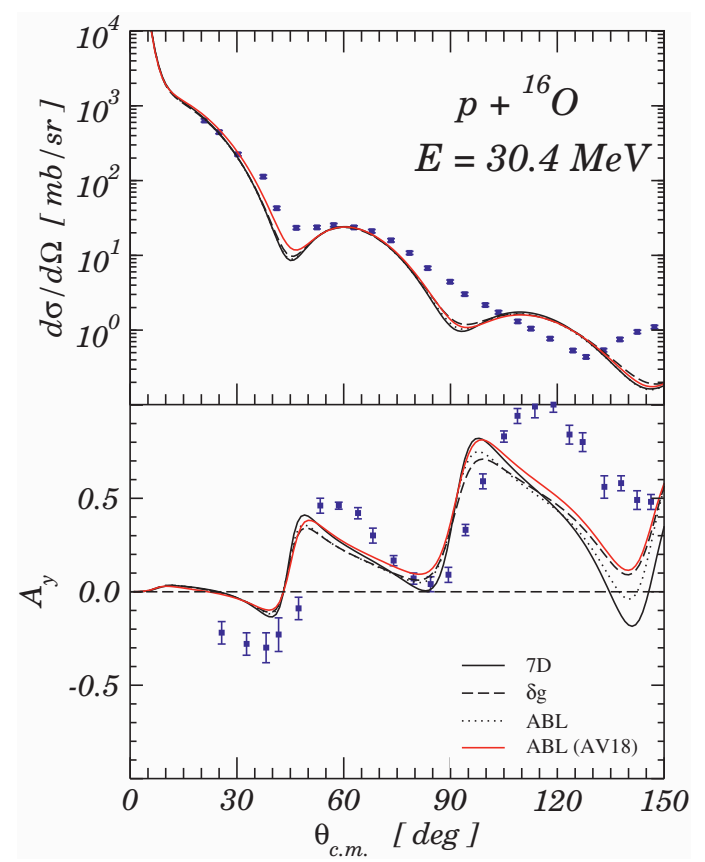

Fig. 1. Measured [11] and calculated differential cross section (upper frame) and analyzing power (lower frame), as functions of the center-of-mass scattering angle, for ${ }^{16} \mathrm{O}(p, p)$ scattering at $30.4 \mathrm{MeV}$. The solid, dashed and dotted curves represent the unabridged, $\delta g$ - and ABL-folding results, respectively. The solid red curves represent ABL results using the Argonne $v_{18}$ potential.

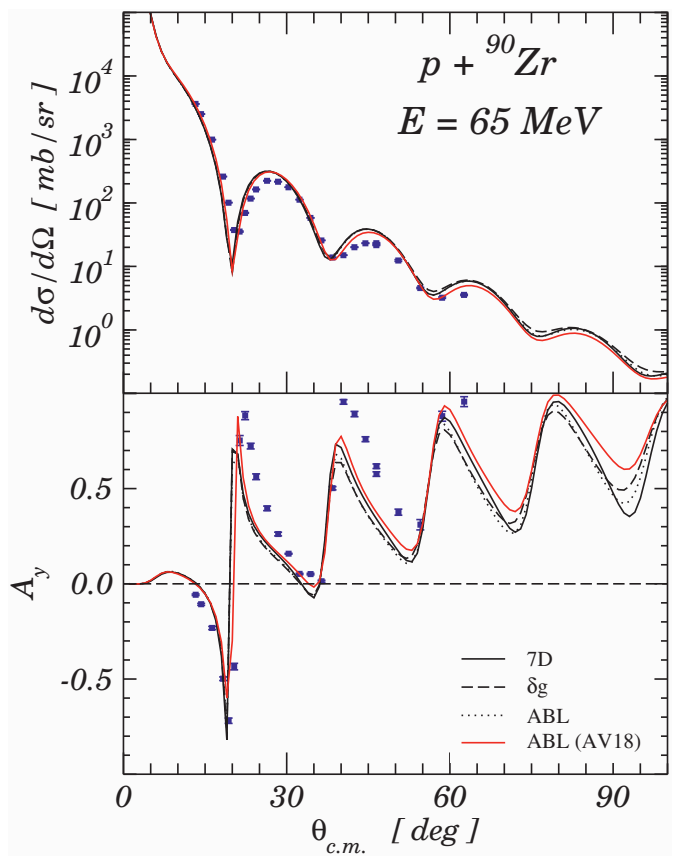

Fig. 2. The same as Fig. (1) but for ${ }^{90} \mathrm{Zr}(\mathrm{p}, \mathrm{p})$ at $65 \mathrm{MeV}$. The data are from Ref. [12]. 


\section{EPJ Web of Conferences}

has been taken as the nuclear matter $g$ matrix from the BBG equation. A comparison with alternative approximations such as the $\delta g$ - or ABL-folding yield marginal differences among the predicted scattering observables. This implies that the off-shell behavior of $g$ matrix weak enough to be insensitive to the non conservation of momentum due to the non-homogeneity of the nucleus. Furthermore, the shortcomings of the folding model cannot be attained to the treatment of the recoil of the target struck nucleon. Not only that, but we have also found that the sensitivity to (phenomenologically) on-shell equivalent $N N$ potentials become more important but also moderate. Even though the 7D-folding is computationally intensive, the ABL approach remains still reliable, with the advantage that it is much less demanding from a computational point of view. With these finding we have set safer grounds from which other effects, at the level of the effective interaction, can be investigated.

H.F.A. acknowledges funding and generous hospitality of CEA/DAM/DIF, Bruyères-le-Châtel, France, during his sabbatical leave where part of this research took place. This work was also funded in part by VID-UCH under grant ENL1106

\section{References}

1. L. Ray, G. W. Hoffmann and W. R. Coker, Phys. Rep. 212, (1992) 223, and references therein.

2. F. A. Brieva and J. R. Rook, Nucl. Phys. A291, (1977) 317; ibid. A307, (1978) 493.

3. H. V. von Geramb, in The Interaction Between Medium Energy Nucleons in Nuclei, edited by $\mathrm{H}$. O. Meyer (AIP, New York,1983).

4. K. Amos, P. J. Dortmans, H. V. von Geramb, S. Karataglidis, and J. Raynal, Adv. in Nucl. Phys. $25,(2000) 275$.

5. H. F. Arellano, F. A. Brieva, and W. G. Love, Phys. Rev. C 52, (1995) 301.

6. F. J. Aguayo and H. F. Arellano, Phys. Rev. C 78, (2008) 014608.

7. Z. H. Li, U. Lombardo, H.-J. Schulze, W. Zuo, L. W. Chen, and H. R. Ma, Phys. Rev. C 74, (2006) 047304.

8. H. F. Arellano and E. Bauge, Phys. Rev. C 84, (2011) 034606.

9. H. F. Arellano and E. Bauge, Phys. Rev. C 76, (2007) 014613.

10. H. F. Arellano and W. G. Love Phys. Rev. C 76, (2007) 014616.

11. P. D. Greaves, V. Hnizdo, J. Lowe and O. Karban, Nucl. Phys. A179, (1972) 1.

12. H. Sakaguchi, M. Nakamura, K. Hatanaka, A. Goto, T. Noro, F. Ohtani, H. Sakamoto, H. Ogawa, and S. Kobayashi, Phys. Rev. C 26, (1982) 944.

13. M. Lacombe, B. Loiseau, J. M. Richard, and R. Vinh Mau, J. Côté, P. Pirés and R. de Tourreil, Phys. Rev. C 21, (1980) 861.

14. R. B. Wiringa, V. G. J. Stoks, and R. Schiavilla, Phys. Rev. C 51, (1995) 38. 